\title{
A novel fractional-order extended Kalman filtering method for on-line joint state estimation and parameter identification of the high power li-ion batteries.
}

CHEN, L., WANG, S., JIANG, H., FERNANDEZ, C. and XIONG, X. 


\title{
A Novel Fractional - Order Extended Kalman Filtering Method for on-line Joint State Estimation and Parameter Identification of the High Power Li-ion Batteries
}

\author{
Lei Chen ${ }^{1}$, Shunli Wang ${ }^{1, *}$, Hong Jiang ${ }^{1}$, Carlos Fernandez ${ }^{2}$, Xin Xiong ${ }^{1}$ \\ ${ }^{1}$ School of Information Engineering, Southwest University of Science and Technology, Mianyang \\ 621010, China; \\ ${ }^{2}$ School of Pharmacy and Life Sciences, Robert Gordon University, Aberdeen AB10-7GJ, UK. \\ "E-mail: $497420789 @$ qq.com
}

Received: 29 December 2020 / Accepted: 16 March 2021 / Published: 31 March 2021

\begin{abstract}
To ensure the reliability and sustainability of the energy storage system, it is important to accurately estimate the state of charge of the battery management system. The Li-ion battery is established based on fractional-order model, and the model parameters are identified online using particle swarm optimization combined with the forgetting factor recursive least square method. On this basis, a novel fractional-order extended Kalman filter method for on-line joint state estimation and parameter identification is proposed. This method can update the parameter model of Li-ion battery in real-time, which not only improves the accuracy of the battery model but also improves the accuracy of SOC estimation. Finally, to verify the accuracy and superiority of the method, the integral order extended Kalman filter, fractional-order extended Kalman filter are compared with the proposed method under the BBDST test schedule. Experimental results show that the algorithm has the highest SOC estimation accuracy and the smallest estimation error $(1.5 \%$.). The results indicate that the fractional-order model can better describe the dynamic characteristics of Li-ion battery, and the adaptive scheme can significantly suppress noise measurement errors and battery model errors. The algorithm realizes online parameter identification and can be used in engineering applications.
\end{abstract}

Keywords: Li-ion battery; fractional-order equivalent circuit model; state of charge; forgetting factor recursive least-square; adaptive fractional-order extended Kalman filter;

\section{INTRODUCTION}

In recent years, to improve the ecological environment and respond to the fossil energy utilization crisis, the development and utilization of renewable energy systems have become very important $[1,2]$. The electric energy storage system has been widely used in electric vehicles, solar energy, wind energy, smart grids, energy internet, and other fields because of its advantages such as auxiliary control of 
renewable energy, reduction of greenhouse gas emissions, fast response speed, and convenient installation [3-5]. It provides a powerful and effective way for the renewable energy system to overcome its inherent weakness of high intermittence and universal randomness [6, 7]. Li-ion battery has become the first choice for energy storage systems in many fields due to its high energy density, long life, safety and reliability, and low environmental pollution $[8,9]$.

The battery management system (BMS) is an important part of the energy storage system, used to manage the operating temperature, current, and terminal voltage of the battery, and prevent the battery from over-discharging and over-charging [10]. As the key part of the BMS, accurate SOC estimation can not only protect the battery from damage but also provide a reference for the reasonable distribution of the BMS [11, 12]. In practical applications, the SOC value cannot be obtained directly, it depends on the real-time terminal voltage and loads current of the battery. Therefore, real-time accurate estimation of SOC is a huge challenge in the complex environment [13].

Equivalent modeling is a necessary condition for accurately estimating the battery SOC, and its accuracy will directly affect the accuracy of SOC [14]. To ensure the reliability and sustainability of the storage system, it is necessary to establish a reliable equivalent model [15]. According to different modeling mechanisms, electrochemical models and equivalent circuit models are commonly used currently[16]. The traditional integer order model (IOM) is widely used because of its simple model. The RC network is used to describe the battery concentration effect, and the number of RC networks will affect the accuracy of the model $[17,18]$. Fractional calculus as an emerging modeling technique has been introduced into battery and ultra-capacitor models [19-21]. The capacitors of existing battery models exhibit fractional-order characteristics [22]. The battery model established by fractional calculus can better describe the dynamic behavior of the RC network and can effectively balance the contradiction between the accuracy of the battery model and the calculation task [23, 24]. The fractional-order model (FOM) uses impedance elements such as constant phase element (CPE) and Warburg element to accurately describe the charge-transfer between solid electrode/electrolyte interface and double layer effect in the electrochemical process, and can better simulate the dynamic characteristics of the system to reduce modeling errors [25].

With the development of the fractional-order theory, the fractional-order Kalman filter (FOKF) has been widely used in practical systems. Scholars have proposed various methods to estimate SOC based on FOKF [26, 27]. Ref.[28] compared five state-of-the-art fractional-order models in terms of SOC estimation, and the parameter identification results show that the increase in complexity does not always improve the accuracy. In [29] [30], Mu et al estimated the SOC of the battery based on FOM and identified the parameters with a bionic optimization algorithm. [31, 32] proposed a FOM containing 2RC networks, which improved the accuracy of the model, but ignored the engineering application, making online parameter identification difficult to achieve. These studies have proved that for different types of batteries, FOM is superior to IOM in terms of voltage simulation and SOC estimation. However, due to the non-linearity of the fractional order, most of the literature uses offline identification methods [33].This method cannot reflect the influence of operating current, charge and discharge status, and temperature on the internal characteristics of the battery under working conditions. Some scholars have realized the importance of online parameter identification [34-36], [37] proposed an online parameter estimation of fractional order system based on gradient algorithm, but it has not been applied to the Li- 
ion battery model. In addition, there are few literatures on joint estimation of parameter identification and SOC estimation[38] [39].

Therefore, to solve the above problems, a novel fractional-order extended Kalman filter method for on-line joint state estimation and parameter identification of the high power Li-ion batteries is proposed to improve the battery management system performance and enhance the reliability and sustainability of the storage system.

\section{THEORY AND METHOD RESEARCH}

\subsection{Fractional calculus}

Three most commonly used fractional calculus are defined: Riemann-Liouville (RL) definition, Grünwald-Letnikov (GL) definition, and Caputo Riemann definition[40]. GL definition can directly discretize fractional differential equations and is the easiest definition to use in digital signal control modeling. Use G-L to define the fractional-order as shown in Eq. (1).

$$
D_{t}^{a} f(t)=\lim _{h \rightarrow 0} h^{-a} \sum_{j=0}^{\left[\left(t-t_{0}\right) / h\right]}(-1)\left(\begin{array}{l}
a \\
j
\end{array}\right) f(t-j h)
$$

Where $D_{t}^{a} f(t)$ stands for an order integral-differential of $\mathrm{f}(\mathrm{t})$, and a is the integral differential order, $\mathrm{h}$ is the sampling interval, $\omega_{j}^{a}=(-1)^{j}\left(\begin{array}{l}a \\ j\end{array}\right)$ represent weighting coefficients., and $\left(\begin{array}{l}a \\ j\end{array}\right)$ means Newton binomial coefficient can be calculated as shown in Eq. (2).

$$
\left(\begin{array}{l}
a \\
j
\end{array}\right)=\left\{\begin{array}{l}
1 \quad j=0 \\
\frac{\mathrm{a}(\mathrm{a}-1) \ldots(\mathrm{a}-\mathrm{j}+1)}{j !} \quad j>0
\end{array}\right.
$$

Where $\left[\left(t-t_{0}\right) / h\right]$ means the integer part of $\left(t-t_{0}\right) / h$. When a $>0$, the equation is fractional derivative; when $\mathrm{a}=0$, the equation is the original equation; when $\mathrm{a}<0$, the equation is fractional integral, and the specific form is defined as shown in Eq. (3).

$$
D_{t}^{a} f(t)= \begin{cases}\lim _{h \rightarrow 0} h^{-a} \sum_{j=0}^{[t / h]}(-1)\left(\begin{array}{l}
a \\
j
\end{array}\right) f(t-j h) & a>0 \\
f(t) & a=0 \\
\lim _{h \rightarrow 0} h^{a} \sum_{j=0}^{[t / h]}(-1){ }^{j}\left(\begin{array}{l}
a \\
j
\end{array}\right) f(t-j h) & a<0\end{cases}
$$

\subsection{Equivalent modeling}

In practical applications, it is a huge challenge to accurately estimate the SOC in real-time, and the establishment of the optimal equivalent model is the prerequisite for accurately estimating the SOC. In the traditional IOM[41], the RC network is used to describe the battery concentration effect. Engineering applications usually use the first-order RC equivalent circuit model, but the low-order RC network will affect the accuracy of the model. 
As capacitors have fractional characteristics, the battery model established by fractional calculus can better describe the dynamic behavior of the RC network and can improve the accuracy of the model when the RC network is limited. Therefore, the establishment of a FOM is shown in Fig. 1[42]. The parallel combination consisting of CPE and resistor is used to accurately describe the charge-transfer between solid electrode/electrolyte interface and double layer effect in the electrochemical process, and improve the accuracy of the first-order RC model.

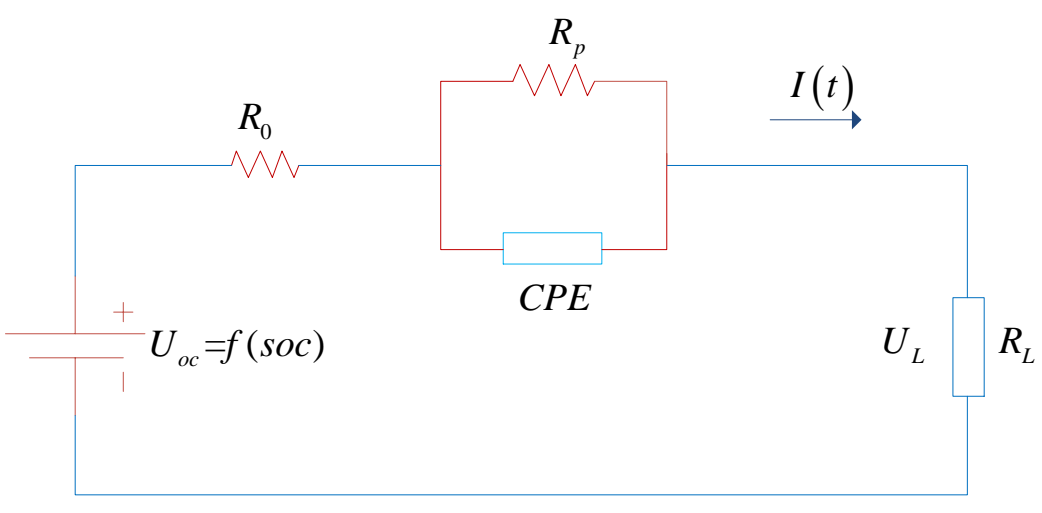

Figure 1. Fractional equivalent circuit model

Where Uoc is the open-circuit voltage, $R_{0}$ is ohmic internal resistance, $R_{\mathrm{P}}$ is polarization resistance, $\mathrm{CPE}$ is a constant phase element, and a is the fractional order of CPE element to describe the dispersion effect. According to the electrochemical principle, the impedance form of CPE is defined as shown in Eq. (4).

$$
Z_{C P E}=\frac{1}{C_{1} S^{a}}
$$

Where $C_{1}$ is the impedance coefficient, according to Kirchhoff's law, the voltage can be expressed as shown in Eq. (5).

$$
\left\{\begin{array}{l}
U_{L}=U_{o c}+U_{0}+U_{p} \\
D^{a} U_{p}=\frac{I}{C_{1}}-\frac{U_{p}}{R_{p} C_{1}}
\end{array}\right.
$$

The SOC is defined as shown in Eq. (6).

$$
S O C_{t}=S O C_{t_{0}}-\frac{\int_{t_{0}}^{t} I(t) \eta d t}{Q}
$$

Based on the definition of G-L calculus, the state space equation is discretized. The operating current $I_{\mathrm{k}}$ is the input of the system, and the discrete state space equation is obtained as shown in Eq. (7).

$$
\left\{\begin{aligned}
{\left[\begin{array}{l}
\operatorname{SOC}(k) \\
U_{p}(k)
\end{array}\right]=\left[\begin{array}{cc}
0 & 1 \\
-\frac{T^{a}}{R_{p} C_{1}} & 0
\end{array}\right]\left[\begin{array}{l}
\operatorname{SOC}(k-1) \\
U_{p}(k-1)
\end{array}\right]+\left[\begin{array}{l}
\frac{\eta T}{Q} \\
\frac{T^{a}}{C_{1}}
\end{array}\right] I_{k}-\left[\begin{array}{c}
0 \\
\lim _{h \rightarrow 0} \sum_{j=1}^{k} \omega_{j}^{a} U_{p}(k-j)
\end{array}\right]+\omega_{k-1} . } \\
U_{L}(k)=U_{O C}-\left[\begin{array}{ll}
0 & 1
\end{array}\right]\left[\operatorname{SOC}(k) \quad U_{p}(k)\right]^{T}-R_{0} I_{k}+v_{k-1}
\end{aligned}\right.
$$




\subsection{Parameter identification}

Particle swarm optimization (PSO) is widely used to solve multi-objective nonlinear optimization problems. This research uses PSO to identify and optimize model parameters to minimize the error between the model terminal voltage and the measured voltage. The main implementation steps are as shown below:

1. The fitness function is defined as shown in Eq. (8).

$$
\text { finess }(\theta)=\min _{\theta} \sum_{k=1}^{N}\left(\left|U_{L}(k)-U_{L}\left(I_{k}, \theta\right)\right|\right)
$$

$U_{L}(k)$ is the measured voltage, and $U_{L}\left(I_{k}, \hat{\theta}\right)$ is the model terminal voltage.

2. The velocity and position of each particle are updated iteratively by formula (9), and the local and global optimal values are updated by calculating the fitness value.

$$
\left\{\begin{array}{l}
v_{k-1}^{i}=\omega^{*} v_{k-1}^{i}+c_{1}|\operatorname{randn}|\left(p_{p b e s t}-x_{k-1}^{i}\right)+c_{2}|\operatorname{randn}|\left(p_{g b e s t}-x_{k-1}^{i}\right) \\
x_{k}^{i}=\mathrm{x}_{k-1}^{i}+v_{k-1}^{i}
\end{array}\right.
$$

3. When the maximum number of iterations is reached or the global optimal position satisfies the minimum limit, the search is stopped and the identification results of the optimal estimation parameters are output.

\section{ADAPTIVE FRACTIONAL - ORDER EXTENDED KALMAN FILTER}

\subsection{Fractional-order extended Kalman filter}

Kalman filtering $(\mathrm{KF})$ and its derivatives are extensively applied in Li-ion battery state estimation under complex operating conditions. It can correct the initial error through the Kalman gain and quickly track the actual SOC during the iterative operation. FO-EKF is derived based on KF and fractional calculus algorithm, mainly including time update and measurement update.

1) Time update:

State vector update: $\quad \bar{x}_{k}=A x_{k-1}+B I_{k-1}-\sum_{j=1}^{k} K_{j} x_{k-j}$

State covariance update: $\bar{P}_{k}=\left(A-K_{1}\right) P_{k-1}\left(A-K_{1}\right)^{T}+Q_{k}+\sum_{j=2}^{k} K_{j} P_{k-j} K_{j}^{T}$

2) Measurement update:

The Kalman gain: $K=\bar{P}_{k} C_{k}^{T}\left(C_{k} \bar{P}_{k} C_{k}^{T}+R_{k}\right)^{-1}$

State vector measurement update: $\hat{x}_{k}=\bar{x}_{k}+K\left[y(k)-\left(C \bar{x}_{k}+E+U_{0}\right)\right]$

Update state covariance matrix: $P_{k}=\left(I-K_{k} C_{k}\right) \bar{P}_{k}$

Fractional calculus is an operator memorized according to the equation of state, which indicates that the predicted state vector at the current time is related to each state in the past. As the amount of historical data involved in the calculation increases, the estimation accuracy will continue to improve, but the computational burden will increase. Therefore, according to the principle of short-term memory, 
it is necessary to find the most suitable memory length. Through experimental methods, it is determined that $\mathrm{L}=70$.

To balance the relationship between calculation accuracy and computational burden, a two-stage method is used to improve the algorithm. Define the improved state vector shown in Eq. (15).

$$
\left\{\begin{array}{lr}
x_{k}=A x_{k-1}+B I_{k-1}+\omega_{k-1}-\sum_{j=1}^{k} K_{j} x_{k-j} & k \leq L \\
x_{k}=A x_{k-1}+B I_{k-1}+\omega_{k-1}-\sum_{j=k-L+1}^{k} K_{j} x_{k-j} & k>L
\end{array}\right.
$$

Similarly, the improved state covariance matrices can be obtained as shown in Eq. (16).

$$
\left\{\begin{array}{l}
P_{k}=\left(A-K_{1}\right) P_{k-1}\left(A-K_{1}\right)^{T}+Q_{k}+\sum_{j=2}^{k} K_{j} P_{k-j} K_{j}^{T} \quad k \leq L \\
P_{k}=\left(A-K_{1}\right) P_{k-1}\left(A-K_{1}\right)^{T}+Q_{k}+\sum_{j=k-L+1}^{k} K_{j} P_{k-j} K_{j}^{T} \quad k>L
\end{array}\right.
$$

When the number of the accumulated state vector is less than the memory length, the calculation is performed based on the actual accumulation items. When the accumulation item is greater than the memory length L, only the number of items in the memory length is used for calculation. The implementation flow chart of the FO-EKF algorithm is shown in Fig. 2.

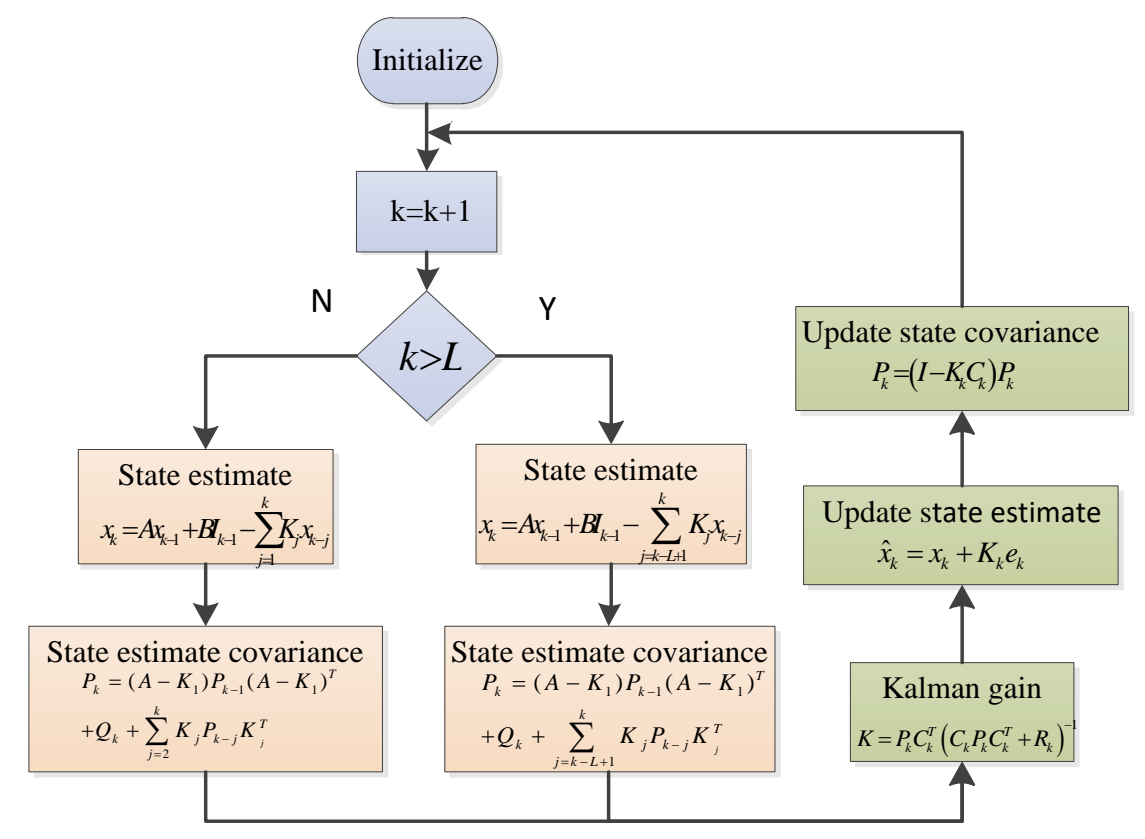

Figure 2. The implementation flow chart of the FO-EKF algorithm

\subsection{Adaptive Fractional-order extended Kalman filter}

The statistical characteristics of FOEKF noise will affect the accuracy of SOC estimation. In practical applications, the noise variance will change as the estimation process changes. A real-time calculation method of covariance matrix based on adaptive fractional-order extended Kalman filter (AFOEKF) is proposed. The method can adjust the noise covariance matrix to make it conform to the 
recursive calculation of residuals, thereby monitoring and tracking the changes of noise sources, and making the system adaptively adjust parameters and estimation accuracy.

The residual $\varepsilon_{k}$ is defined as the difference between the measurement and the predicted output based on the state estimate after measurement update, $\Delta x_{i}$ is defined as the difference between the state estimate measurement and the time domain update measurement. Where $R_{\varepsilon k}$ and $R_{\Delta x}$ are the statistical sample variance estimate. $\mathrm{M}$ represents the adaptive smoothing filter window.

$$
\left\{\begin{array}{l}
\varepsilon_{k}=y_{k}-\left(C x_{k}+E+U_{0}\right) \\
R_{\varepsilon k}=\frac{1}{M} \sum_{i=k-M+1}^{k} \varepsilon_{i} \varepsilon_{i}^{T}
\end{array}\right.
$$

The measurement noise and process noise can be calculated as shown in Eq. (18).

$$
\begin{aligned}
& R=R_{\varepsilon k}-C_{k} P_{k} C_{k}^{T} \\
& Q_{k}=P_{k}-\left(A-K_{1}\right) P_{k-1}\left(A-K_{1}\right)^{T}+\sum_{j=2}^{k} K_{j} P_{k-j} K_{j}^{T}+R_{\Delta x} \\
& R_{\Delta x}=\frac{1}{M} \sum_{i=k-M+1}^{k} \Delta x_{i} \Delta x_{i}^{T}
\end{aligned}
$$

\section{A NOVEL ON-LINE JOINT ESTIMATION METHOD OF SOC AND FRACTIONAL ORDER MODEL PARAMETER IDENTIFICATION}

\subsection{Forgetting factor recursive least square method}

Online parameter identification is necessary because the parameters will be affected by SOC, ambient temperature, battery aging, and other factors, and are closely related to the operating state of the battery. If the PSO algorithm has identified the fractional order, then the remaining parameters can be identified by the FFRLS algorithm.

Eq. (19) is the transfer function of the model impedance:

$$
\frac{U_{O C}(s)-U_{0}(s)}{I(s)}=\frac{R_{p}}{R_{p} C_{1} s^{a}+1}+R_{0}
$$

According to the G-L definition, the above equation can be discretized as shown in Eq. (20).

$$
\left\{\begin{array}{l}
y(t)+R_{p} C_{1} T^{-a} \sum_{i=0}^{N}(-1)^{i}\left(\begin{array}{c}
a \\
i
\end{array}\right) y(t-i T)=I(t)\left(R_{0}+R_{p}\right)+R_{0} R_{p} C_{1} T^{-a} \sum_{i=0}^{N}(-1)^{i}\left(\begin{array}{l}
a \\
i
\end{array}\right) I(t-i T) \\
A(i)=T^{-a}\left(\begin{array}{c}
a \\
i
\end{array}\right) y(t-i T)
\end{array}\right.
$$

Considering the accuracy requirements of the battery model, the memory length is truncated and converted into a first-order difference equation.

$$
y(k)=-\omega_{1}^{a} \frac{m}{T^{a}+m} y(k-1)+\left(\frac{T^{a} R_{p}}{T^{a}+m}+R_{0}\right) I(k)+\omega_{1}^{a} \frac{m R_{0}}{T^{a}+m} I(k-1)
$$

Where $\mathrm{m}=R_{p} C_{1}$, and the model parameters can be identified as shown in Eq. (22). 


$$
\left\{\begin{array}{l}
R_{0}=a_{3} / a_{1} \\
m=a_{1} T^{a} /\left(a-a_{1}\right) \\
R_{p}=\left(a_{2}-R_{0}\right)\left(T^{a}+m\right) / T^{a} \\
C_{1}=m / R_{p}
\end{array}\right.
$$

\subsection{A new method of fractional order parameter identification and SOC online joint estimation}

The online parameter identification is more suitable for engineering because the battery parameters change with SOC in practical application. In addition, the online joint estimation method of ECM parameters and SOC has been applied in many fields[43, 44]. However, online joint estimation methods based on FOM parameters and SOC are rarely implemented[45], and the influence of actual environment noise is not considered.

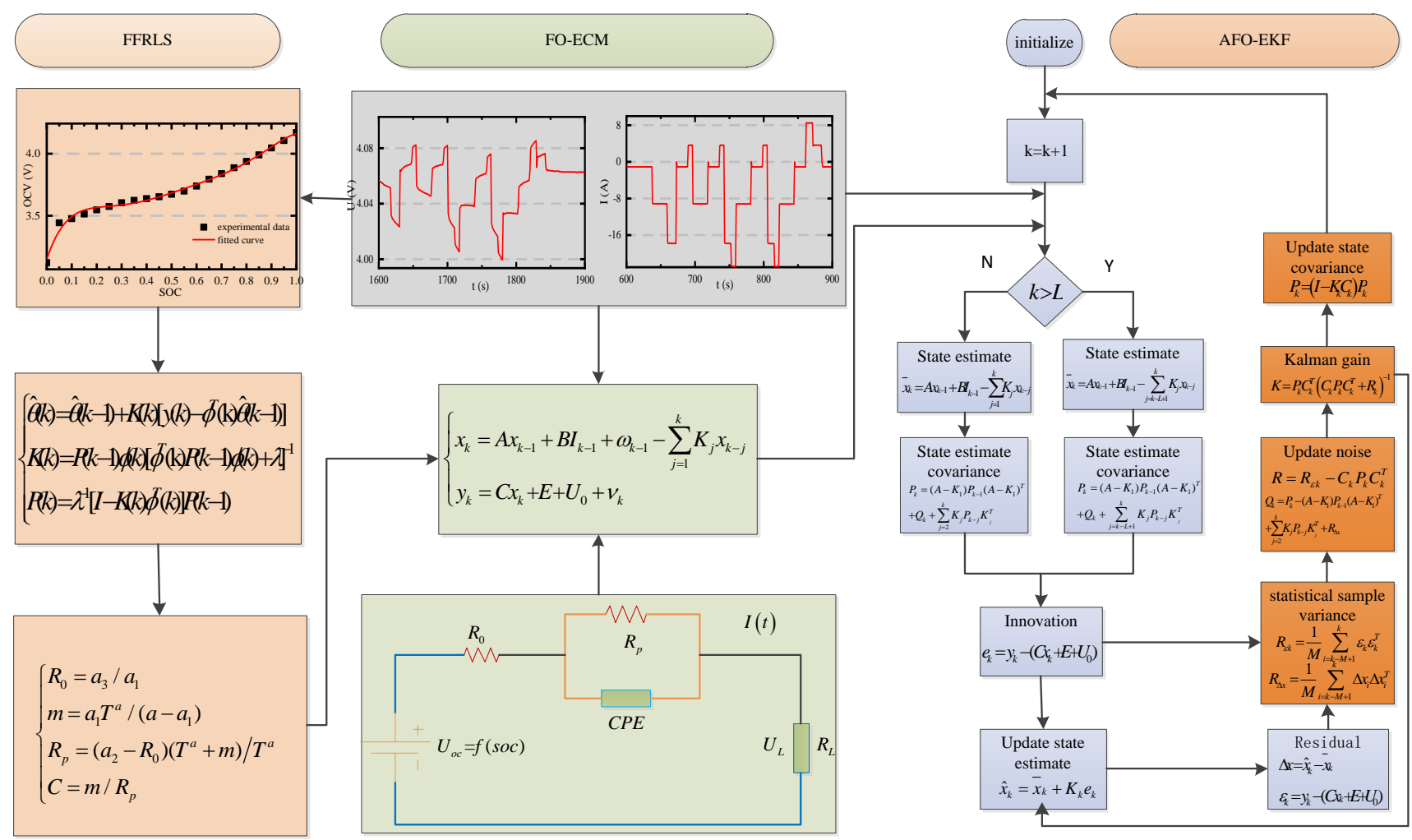

Figure 3. The implementation flowchart of the Online-AFOEKF algorithm

An online joint estimation method of parameter identification and adaptive extended Kalman filter is proposed (Online-AFOEKF), which is used to estimate the SOC of Li-ion battery based on FOM. The implementation flow chart of the AFOEKF algorithm is shown in Fig. 3. The joint estimation of SOC and parameters can update parameters in real-time, and track and adjust the noise covariance matrix through AEKF, which can effectively suppress the influence of noise and improve the convergence speed of the algorithm. The FFRLS algorithm is used to identify the main parameters online, and then the 
AEKF algorithm is used to estimate the battery of SOC based on the FOM. The parameters and SOC are updated iteratively over time to achieve the optimal estimation.

\section{EXPERIMENTAL ANALYSIS}

\subsection{Experimental working conditions}

The experimental equipment includes ternary Li-ion battery, the battery testing equipment (CT4016-5V100A-NTFA), the temperature box (DGBELL BTT-331C), and a computer for humancomputer interaction. The voltage and current curves during the BBDST test are shown in Fig.4 (a) and (b), respectively. A complete BBDST cycle lasts for 300s. Fig.4 (c) and (d) are the voltage and current curves during the BBDST test of one cycle respectively.

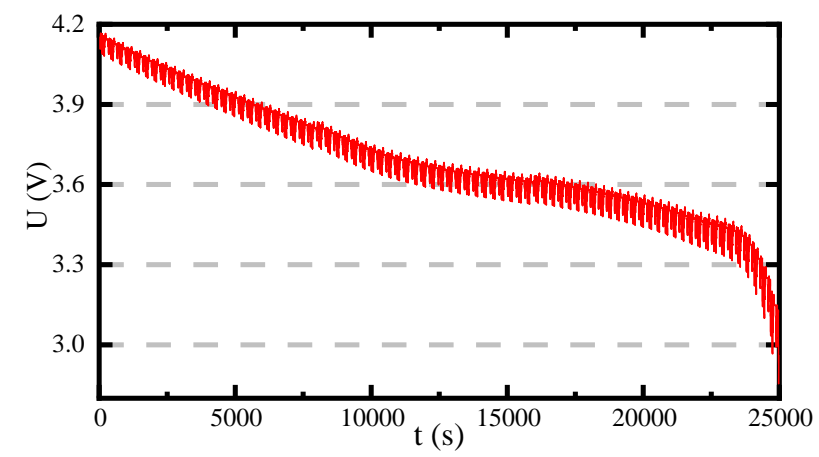

(a) Full cycle voltage

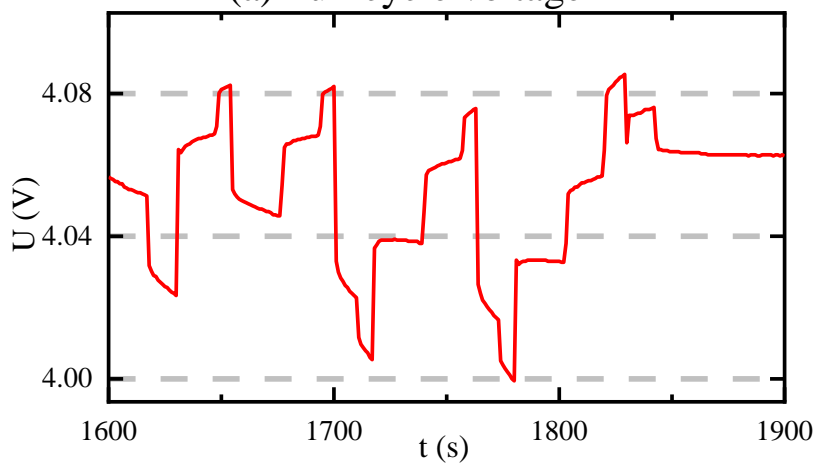

(c) Single period voltage

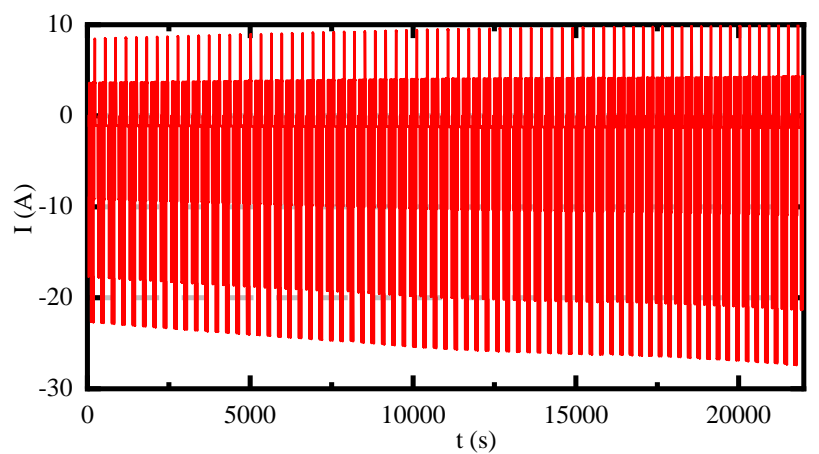

(b) Full periodic current

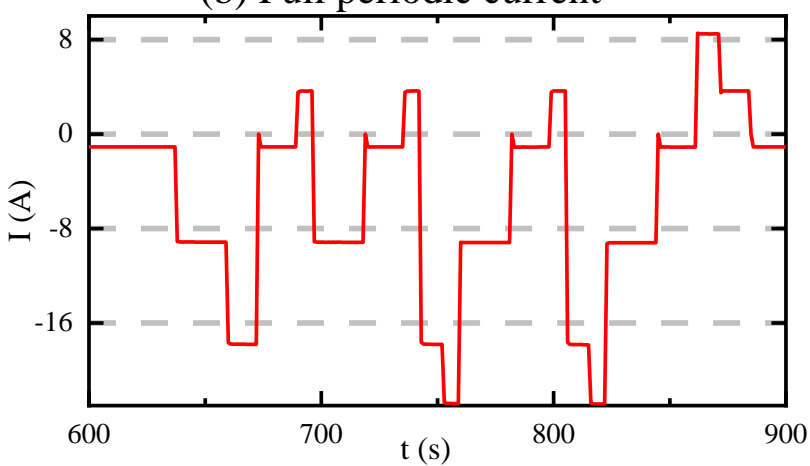

(d) Single period current

Figure 4. experimental data of BBDST condition

\subsection{Parameter identification results}

\subsubsection{OCV identification results}

The relationship between OCV and SOC is established based on experimental data. First, the battery is charged with constant-voltage and the cut-off voltage is $4.2 \mathrm{~V}$. The battery is continuously discharged and placed aside at a discharge rate of $0.3 \mathrm{C}$, and the open-circuit voltage is sampled according 
to the experimental data when the battery is placed.

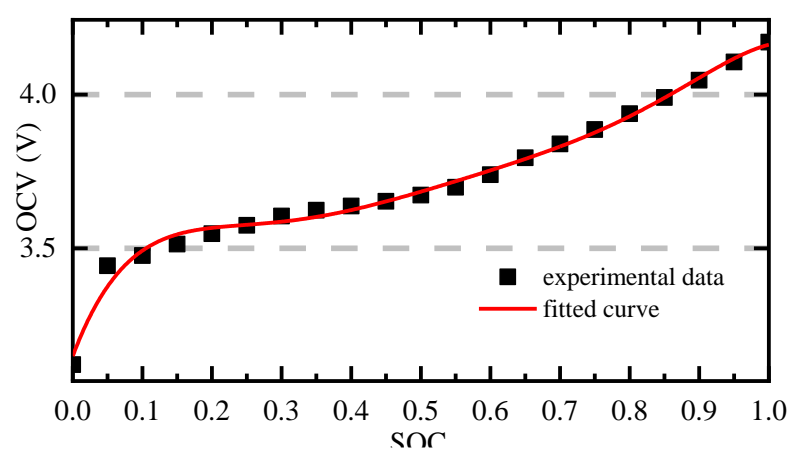

Figure 5. The ocv-soc fitted curve

The OCV-SOC curve can be fitted by the sixth-order polynomial form, and the fitted curve is described as shown in Fig. 5. The fitted coefficients are described as shown in Table 1.

Table 1. The fitting coefficient of ocv-soc

\begin{tabular}{lllllll}
\hline K1 & K2 & K3 & K4 & K5 & K6 & K7 \\
\hline-33.276 & 112.60 & -150.55 & 100.99 & -34.79 & 6.04 & 3.149 \\
\hline
\end{tabular}

\subsubsection{Parameter identification results based on PSO}

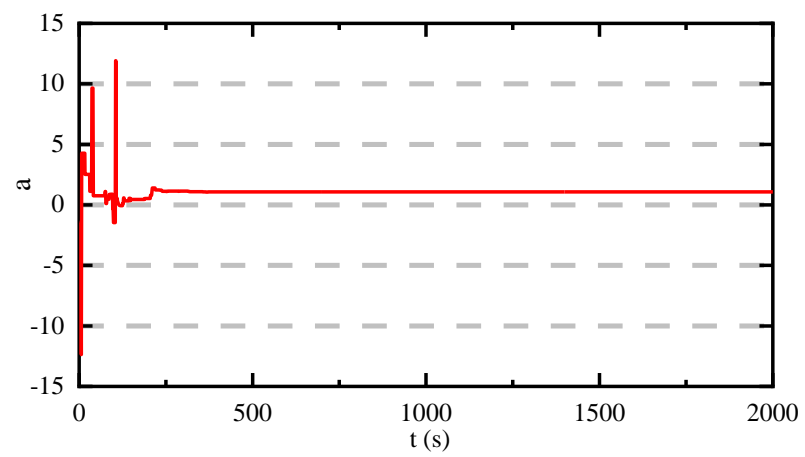

(a) The identification result of a

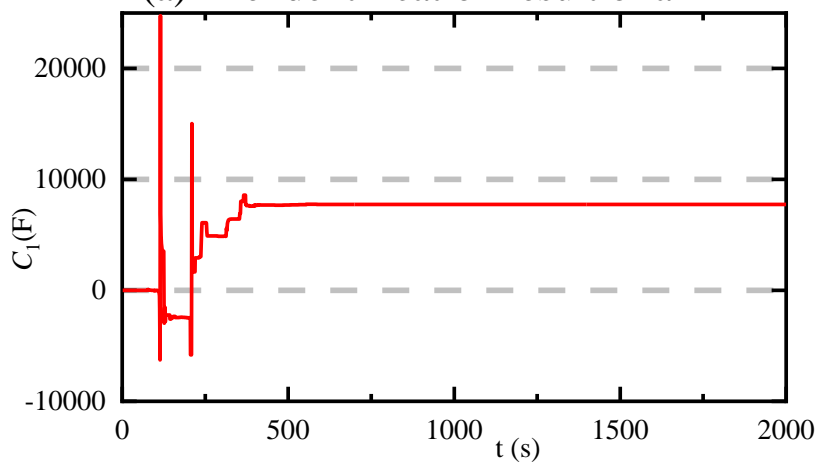

(c) The identification result of $C_{1}$

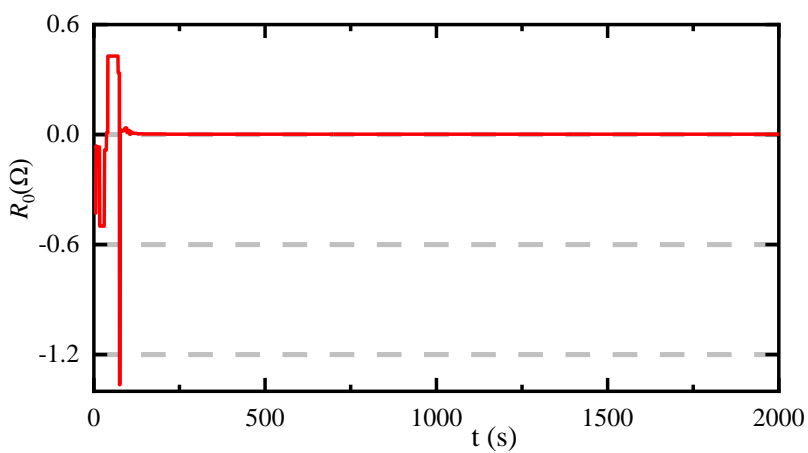

(b) The identification result of $R_{0}$

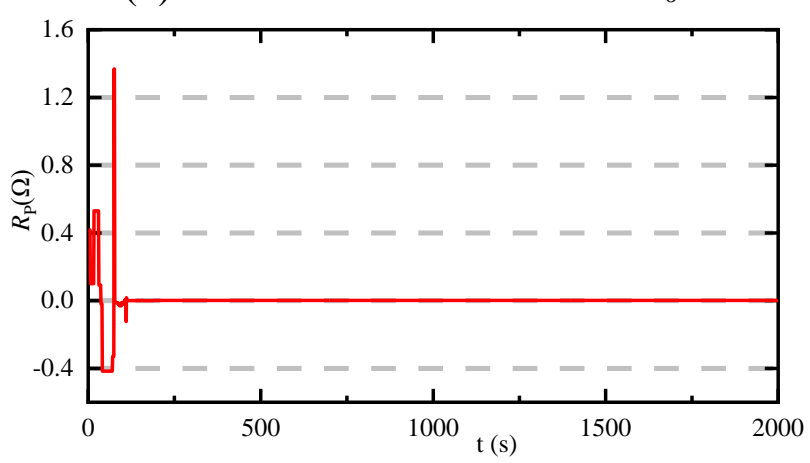

(d) The identification result of $R_{\mathrm{p}}$

Figure 6. Offline identification results of fractional order parameters based on PSO 
The parameters of FOM are identified by the PSO algorithm, and the number of iterations is set to 2000 times. Fig. 6 (a), (b), (c), and (d) represent the results of a, $R_{0}, C_{1}$ and $R_{\mathrm{p}}$ respectively. The figure shows that the identification results can converge in a very short time when the initial value is not accurate.

\subsubsection{Parameter identification results based on FFRLS}

According to the real-time current and voltage data recorded by the BBDST test experiment, the identification results of $R_{0}, C_{1}$, and $R_{\mathrm{p}}$ are obtained as shown in Fig. 7. The current data are used as the input to the model for parameter identification, and the resulting model parameter identification outcomes are obtained based on the FFRLS algorithm.

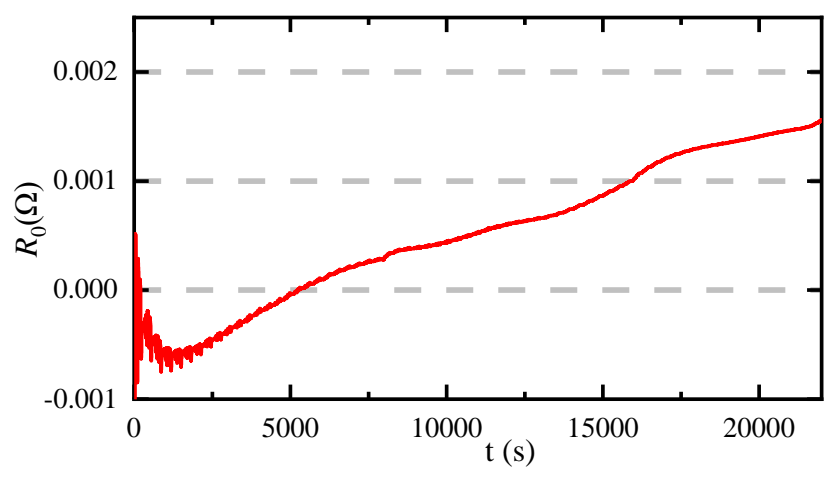

(a) The identification result of $R_{0}$

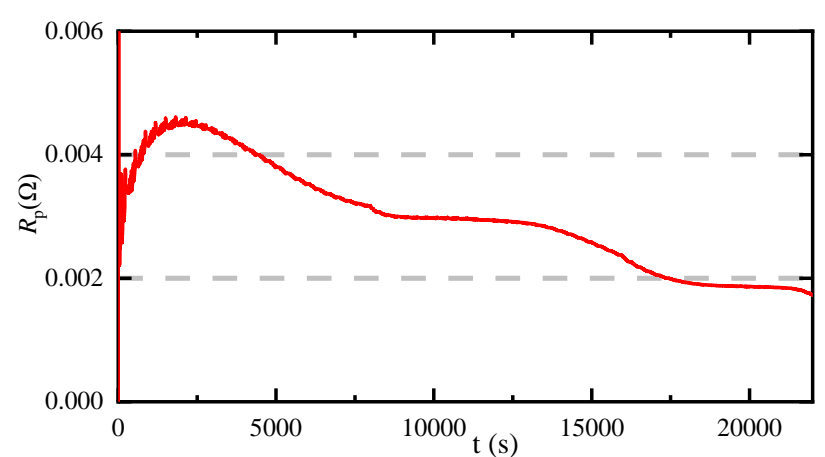

(b) The identification result of $R_{\mathrm{p}}$

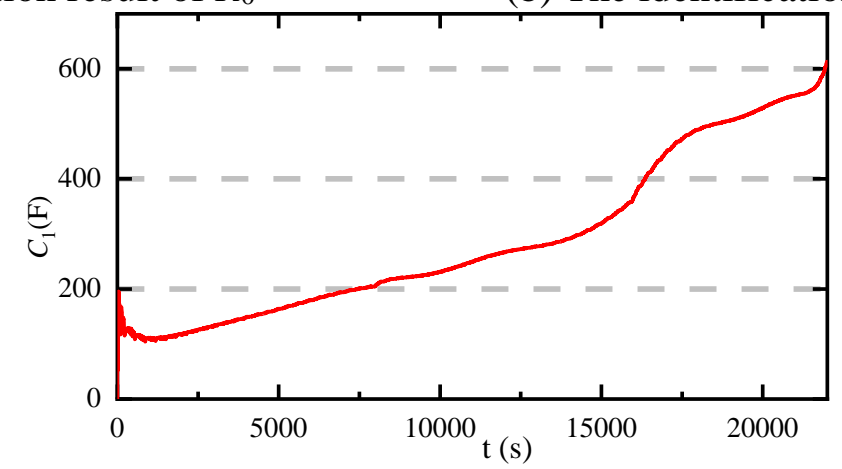

(c) The identification result of $C_{1}$

Figure 7. Online identification results of fractional order parameters based on FFRLS

\subsection{State of charge estimation results and verification}

\subsubsection{Verification of model accuracy}

To verify the FOM results under BBDST conditions, the current data is taken as the model input, and the model voltage output is obtained. The comparison between the model output voltage and the 
measured voltage is shown in Fig 8(a). Fig. 8(b) is the error diagram of the model output voltage and the measured voltage.

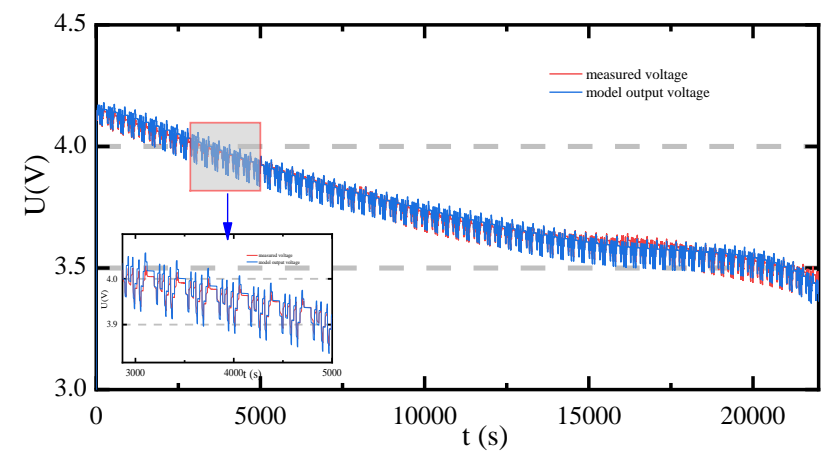

(a) Comparison voltage between the model output and the measured

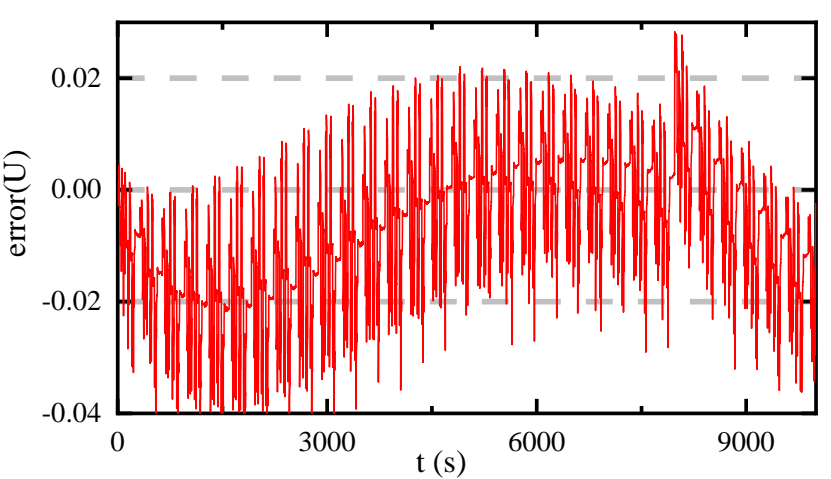

(b) Error between the model output voltage and the measured voltage

Figure 8. Accuracy verification diagram of BBDST condition

The figure shows that the model output voltage and the measured voltage curve have a high degree of fit, and its value is very close to the measured voltage, indicating that the accuracy of parameter identification is high. The estimated RMSE is 0.0144 , and the error between the model voltage and the measured voltage is basically within $0.02 \mathrm{~V}$, which verifies the high accuracy of the proposed model.

\subsubsection{Verification of SOC estimation accuracy}

To verify the accuracy and superiority of the proposed method, the integral order EKF (IOEKF), Offline/Online FOEKF, AFOEKF are compared with the method. The root mean square error (RMSE), mean absolute error (MAE), and mean absolute percentage error (MAPE) are used as indicators to verify the effectiveness of the four SOC estimation algorithms. The results are described as shown in Table 2.

Table 2. SOC performance comparison of four algorithms

\begin{tabular}{ccccccc}
\hline & \multicolumn{2}{c}{$\begin{array}{c}\text { Comparison of battery } \\
\text { models }\end{array}$} & \multicolumn{2}{c}{$\begin{array}{c}\text { Comparison of } \\
\text { parameter } \\
\text { identification results }\end{array}$} & \multicolumn{2}{c}{$\begin{array}{c}\text { Comparison of noise } \\
\text { characteristics }\end{array}$} \\
\cline { 2 - 7 } & Offline & Offline & Offline & Online & Online & Online \\
RMSE & IOEKF & FOEKF & FOEKF & FOEKF & FOEKF & AFOEKF \\
\cline { 2 - 7 } MAPE & $1.53 \%$ & $2.63 \%$ & $2.63 \%$ & $2.34 \%$ & $2.34 \%$ & $1.5 \%$ \\
MAE & $10.14 \%$ & $5.99 \%$ & $5.99 \%$ & $5.53 \%$ & $5.53 \%$ & $3.37 \%$ \\
\hline
\end{tabular}

Table 2 shows that the RMSE, MAPE, and MAE of Online-AFOEKF are 1.5\%, 3.37\%, and 1.2\%, 
respectively. The AFOEKF algorithm proposed in the literature[46] has a smaller average error of less than $1.6 \%$ compared with FO-EKF and IO-AEKF. Since the article is based on online parameter identification, its accuracy will be higher. The results show that FOM has a higher model accuracy than IOM, which improves the accuracy of SOC estimation results. Fig. 9 shows a comparison of SOC estimation based on four algorithms.

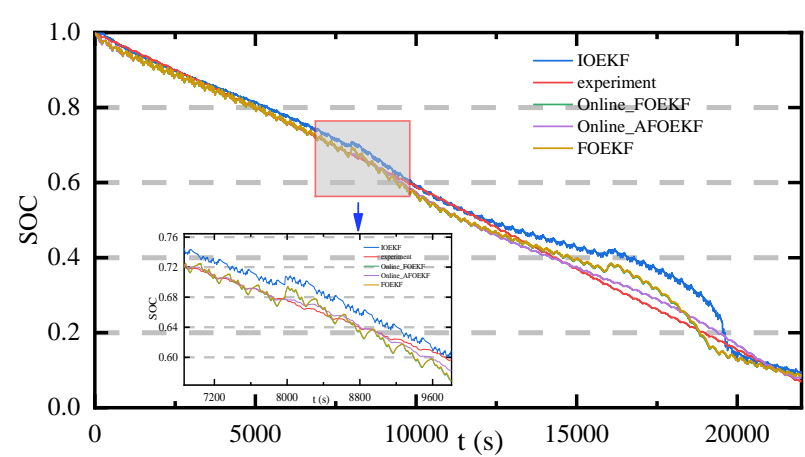

(a) SOC estimation comparison based on four algorithms

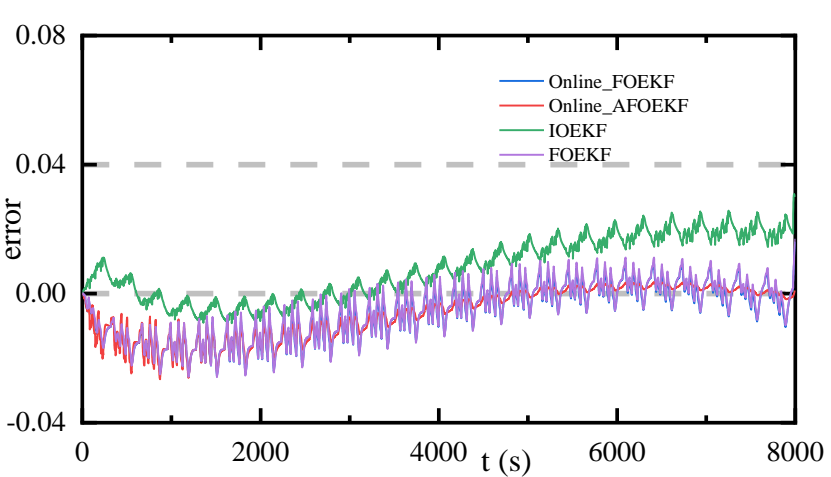

(b) SOC error comparison based on four algorithms

Figure 9. SOC estimation comparison based on four algorithms

(1) Influence of battery model accuracy on SOC

Considering that the accuracy of the battery model varies with the SOC, IOM and FOM are used for equivalent modeling, the EKF method is used to obtain the SOC, and the PSO algorithm is used for model parameter identification. Fig 9 shows the SOC estimation results and error results based on FOM and IOM in the entire SOC range under the BBDST test schedule. The RMSE of FOM and IOM are $2.63 \%$ and $4.53 \%$ respectively, which indicates that the accuracy of SOC estimation based on FOM is much higher than that based on IOM, especially in the low SOC range, because FOM can better describe the dynamic characteristics of the battery.

(2) Influence of different parameter identification methods on SOC

Considering that the accuracy of different parameter identification methods varies with the SOC, Online-FOEKF, and Offline-FOEKF are used to estimate the SOC. First use FFRLS to update the model parameters online, and obtain real-time SOC according to FOEKF. Fig 9(a) shows the RMSE of onlineFOEKF is $2.34 \%$, which proves that on-line identification of model parameters helps improve the adaptability of the model to current working conditions and ambient temperature, and plays an important role in improving model accuracy.

(3) Influence of noise statistical characteristics on SOC

Considering that the accuracy of the noise statistical characteristics varies with the SOC, FOEKF, and AFOEKF are used to estimate SOC respectively. Both of these algorithms are based on FOM, and a noise adaptive algorithm is added to AFOEKF. Fig 9 shows the SOC estimation results and error results based on FOEKF and AFOEKF. The RMSE of FOEKF and AFOEKF are $2.34 \%$ and $1.5 \%$ respectively, 
indicating that the noise adaptive algorithm can significantly suppress the interference of EKF estimation errors, improve the estimated accuracy of SOC, and reflect the advantages of the adaptive scheme.

The results show that the algorithm has high SOC accuracy and can quickly converge to the true value under dynamic conditions, which proves that the algorithm has good robustness and stability.

\section{CONCLUSIONS}

To improve the performance of the battery management system and enhance the reliability and sustainability of the energy storage system, a novel online joint state estimation and parameter identification method for Li-ion battery based on adaptive fractional-order extended Kalman filtering is proposed. The FOM is introduced to describe the dynamic characteristics of the battery, and the parameters of the battery model are updated by combining the PSO algorithm and the FFRLS method. The proposed joint estimation method not only improves the accuracy of the battery model, but also improves the accuracy of the SOC. At the same time, the algorithm can suppress noise errors and battery model errors. The estimation error of the proposed SOC estimation algorithm is less than $1.5 \%$ under the BBDST condition. The results show that the algorithm has high robustness and stability while improving the accuracy of SOC estimation. The algorithm realizes the online parameter identification and can be applied to engineering applications.

\section{ACKNOWLEDGEMENTS}

This research was supported by National Natural Science Foundation (No. 61801407), Sichuan Province Science and Technology Support Program (No. 19ZDYF1098, 2018GZ0390, 2017FZ0013), Scientific Research Fund of Sichuan Provincial Education Department (No. 17ZB0453). Thanks to the sponsors.

\section{References}

1. J. Tian, R. Xiong, W. Shen and F. Sun, Science China-Technological Sciences, (2020).

2. L. Wang, Y. Wang, C. Liu, D. Yang and Z. Chen, IEEE Transactions on Power Electronics, 35 (2020) 5897.

3. Y. Wang, J. Tian, Z. Sun, L. Wang, R. Xu, M. Li and Z. Chen, Renewable \& Sustainable Energy Reviews, 131 (2020).

4. X. Hu, H. Yuan, C. Zou, Z. Li and L. Zhang, IEEE Trans. Veh. Technol., 67 (2018) 10319.

5. C. Zou, L. Zhang, X. Hu, Z. Wang, T. Wik and M. Pecht, J. Power Sources, 390 (2018) 286.

6. A. Nasser-Eddine, B. Huard, J.-D. Gabano and T. Poinot, Control Engineering Practice, 86 (2019) 96.

7. C. Liu, Y. Wang and Z. Chen, Energy, 166 (2019) 796.

8. $\quad$ Y. Wang, X. Zhang, C. Liu, R. Pan and Z. Chen, J. Power Sources, 389 (2018) 93.

9. B. Yang, J. Wang, X. Zhang, J. Wang, H. Shu, S. Li, T. He, C. Lan and T. Yu, J. Power Sources, 448 (2020) 227444.

10. S.-L. Wang, C. Fernandez, C.-Y. Zou, C.-M. Yu, L. Chen and L. Zhang, Energy, 171 (2019) 444.

11. J. Peng, J. Luo, H. He and B. Lu, Appl. Energy, 253 (2019) 113520.

12. N. Chen, P. Zhang, J. Dai and W. Gui, IEEE Access, 8 (2020) 26872.

13. B. Xia, S. Guo, W. Wang, Y. Lai, H. Wang, M. Wang and W. Zheng, Energies, 11 (2018). 
14. X. Lai, L. He, S. Wang, L. Zhou, Y. Zhang, T. Sun and Y. Zheng, Journal of Cleaner Production, 255 (2020).

15. C. Zou, X. Hu, S. Dey, L. Zhang and X. Tang, IEEE Trans. Ind. Electron., 65 (2018) 5951.

16. M. Yu, Y. Li, I. Podlubny, F. Gong, Y. Sun, Q. Zhang, Y. Shang, B. Duan and C. Zhang, Journal of Advanced Research, 25 (2020) 49.

17. Q. Zhang, Y. Shang, Y. Li, N. Cui, B. Duan and C. Zhang, ISA Trans, 97 (2020) 448.

18. J. Tian, R. Xiong and Q. Yu, IEEE Trans. Ind. Electron., 66 (2019) 1576.

19. Q. Yang, J. Xu, X. Li, D. Xu and B. Cao, Int. J. Electr. Power Energy Syst., 119 (2020).

20. $\quad$ R. Xiong, J. Tian, W. Shen and F. Sun, IEEE Trans. Veh. Technol., 68 (2019) 4130.

21. L. De Sutter, Y. Firouz, J. De Hoog, N. Omar and J. Van Mierlo, Electrochim. Acta, 305 (2019) 24.

22. Y. Wang, Y. Chen and X. Liao, Fractional Calculus and Applied Analysis, 22 (2019) 1449.

23. Y. Wang, G. Gao, X. Li and Z. Chen, J. Power Sources, 449 (2020) 227543.

24. C. Liu, W. Liu, L. Wang, G. Hu, L. Ma and B. Ren, J. Power Sources, 320 (2016) 1.

25. X. Lu, H. Li and N. Chen, Electrochim. Acta, 299 (2019) 378.

26. K. S. R. Mawonou, A. Eddahech, D. Dumur, D. Beauvois and E. Godoy, J. Power Sources, 435 (2019).

27. S. Liu, X. Dong and Y. Zhang, IEEE Access, 7 (2019) 122949.

28. J. Tian, R. Xiong, W. Shen and J. Wang, Chinese Journal of Mechanical Engineering, 33 (2020).

29. H. Mu, R. Xiong, H. Zheng, Y. Chang and Z. Chen, Appl. Energy, 207 (2017) 384.

30. J. I. Hidalgo-Reyes, J. F. Gomez-Aguilar, R. F. Escobar-Jimenez, V. M. Alvarado-Martinez and M. G. Lopez-Lopez, Microelectronics Journal, 85 (2019) 109.

31. M. Hu, Y. Li, S. Li, C. Fu, D. Qin and Z. Li, Energy, 165 (2018) 153.

32. G. Jin, L. Li, Y. Xu, M. Hu, C. Fu and D. Qin, Energies, 13 (2020).

33. L. Zhang, S. Wang, D.-I. Stroe, C. Zou, C. Fernandez and C. Yu, Energies, 13 (2020) 2057.

34. L. Li, H. Zhu, A. Zhou, M. Hu, C. Fu and D. Qin, Int. J. Electrochem. Sci., 15 (2020) 6863.

35. X. Liu, C. Zheng, J. Wu, J. Meng, D.-I. Stroe and J. Chen, Energies, 13 (2020).

36. C. Unterrieder, C. Zhang, M. Lunglmayr, R. Priewasser, S. Marsili and M. Huemer, J. Power Sources, 278 (2015) 274.

37. Y. Wei, Z. Sun, Y. Hu and Y. Wang, Journal of Control and Decision, 2 (2015) 219.

38. Y. Xu, M. Hu, A. Zhou, Y. Li, S. Li, C. Fu and C. Gong, Applied Mathematical Modelling, 77 (2020) 1255.

39. J. Tian, R. Xiong, W. Shen, J. Wang and R. Yang, Journal of Cleaner Production, 247 (2020).

40. Q. Zhang, Y. Li, Y. Shang, B. Duan, N. Cui and C. Zhang, Electronics, 8 (2019).

41. X. Li, Z. Wang and L. Zhang, Energy, 174 (2019) 33.

42. J. Wang, L. Zhang, D. Xu, P. Zhang and G. Zhang, Math. Prob. Eng., 2019 (2019) 1.

43. B. Xia, R. Huang, Z. Lao, R. Zhang, Y. Lai, W. Zheng, H. Wang, W. Wang and M. Wang, Energies, 11 (2018).

44. W. Xu, J. Xu and X. Yan, J. Power Electron., 20 (2020) 292.

45. J. Tian, R. Xiong, W. Shen and J. Wang, Energy, 176 (2019) 874.

46. Q. Zhu, M. Xu, W. Liu and M. Zheng, Energy, 187 (2019).

(C) 2021 The Authors. Published by ESG (www.electrochemsci.org). This article is an open access article distributed under the terms and conditions of the Creative Commons Attribution license (http://creativecommons.org/licenses/by/4.0/). 